nuclear-weapons programme, announced that it would like to reduce costs by moving nearly all its plutonium into a single facility. The consolidated plutonium centre, as it would be called, would be built at a pre-existing site and would place plutonium-related research, surveillance and manufacturing under a single roof.

That would be a positive move. Plutonium is too widely dispersed under the current arrangement, with significant quantities being held near populated areas, such as Livermore in California. Confining plutonium to a single, remote site therefore makes sense - as do elements of the plan that would consolidate existing high-explosive and hydrodynamic testing facilities.

Other aspects of the plan are more troubling, however. Specifically, the plutonium centre would be equipped to produce around 125 new plutonium 'pits' per year - these are the cores of modern nuclear weapons. NNSA officials say that the new production capability is necessary to help build a "reliable replacement warhead" - a new kind of warhead that is supposed to require less maintenance than existing designs (see Nature 442, 18-21; 2006). They argue that these warheads would replace, rather than augment, the existing stockpile.

But the construction of such a facility is liable to prompt other nations to revisit their own production plans. Although it won't change the course of countries such as Iran, it reinforces the increasingly prevalent view that nuclear weapons are vital to any nation's security. That perception may encourage further development in non-nuclear states, such as Japan and Brazil. It also sends a dear message to the United States' old nemesis Russia, which continues to maintain an unnecessarily large nuclear stockpile of its own.

The United States has not yet made a convincing case that the new warheads are needed - tests on existing plutonium pits suggest that they will last for several decades, and the replacement warhead is in any case still in the design phase. What's more, the United States already has a working pit facility at Los Alamos National Laboratory in New Mexico, which some specialists think could be adapted to produce some 50 pits a year.

Increasing pit production and building new kinds of weapons run contrary to the spirit of the 1970 Nuclear Non-Proliferation Treaty, which calls for existing nuclear-weapons states to take steps towards disarmament. Instead, the United States and other nuclear states should be acting to shrink both their weapons stockpiles and their production complexes. The best thing they could do for their collective future security would be to demonstrate to the world that nuclear weapons are less central to their own defence strategies than they were during the cold war.

\section{Plan bee}

\section{Another day, another genome.}

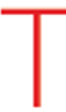
oday, Nature publishes a genome sequence and analysis of the western honeybee, Apis mellifera (see pages 893, 919 and 931). But hasn't this genome lark become a bit ho-hum? From dogs to trees to microbes and, of course, people, the US National Human Genome Research Institute lists more than 50 genome projects either complete or under way. The publication of a genome sequence used to be so exciting. Is it now destined to be dull?

Not if supporters of the honeybee project have anything to say about it. Two other insects have alreadybeen sequenced: the malariacarrying mosquito Anopheles gambiae, and one of science's great model organisms, the fruitfly Drosophila melanogaster. Like these, the bee is much easier to manipulate and study than, say, the monkey. But unlike the mosquito and the fruitfly, the bee's social behaviour is of special interest.

Many schoolchildren learn about the waggle dance, the remarkable figure-of-eight movement that bees use to pass on the location of food. And children aren't the only ones entranced by the hierarchy ofbees. Every bee has its place, from the nurses who tend the young to the foragers who collect food. There is something amazing about this insect's ability to self-organize into a productive, harmonious unit.

Indeed, the bee may fascinate us precisely because of the apparent parallels between bee society and our own - from the humble worker to the regal queen. Bee jobs are highly specialized: there are forager bees, for instance, who only scout for food, and those who follow the scouts' lead once food is found. There are bees who prefer to gather nectar and others who choose pollen. There are even designated undertaker bees, who remove their dead comrades from the colony. This tidy division of labour mirrors what we see in our own species every day. And in perhaps the strongest parallel of all, the bee hierarchy sometimes breaks down entirely - a situation aptly termed 'anarchy'.

It is tempting to wonder whether the mechanisms controlling such complex bee behaviour are related to those at work in human society. The bee genome may allow us to get at the roots of social behaviour that is similar to our own. Researchers cannot engineer human society, by separating families, for example, or setting up entire communities in which every inhabitant is the same age. But they can do this in bees - and then measure the effects of these interventions on bee genes and physiology. And that can help elucidate the environmental, genetic and social roots of bee behaviour. Herein lies the utility of the bee: it lies somewhere between the human and the fruitfly. Like us, it is both complex and familiar. But like a fruitfly, it is easy to study in controlled conditions.

Of course, the comparison between

"There is something
amazing about
this insect's ability
to self-organize
into a productive,
harmonious unit."
bees and humans can only go so far. Bee individuals in a colony are more closely related than people in most cities or towns. And although there are some genetic and mechanical similarities between people and bees, we are vastly different creatures. We don't know what drives most bee or human behaviours, so it's anyone's guess whether they're at all related. But for the community of bee researchers who study this insect purely because it is an intriguing animal in its own right, that doesn't really matter. Regardless of whether the bee tells us anything about ourselves, it fully deserves its moment in the sun. 